\title{
Sex differences in cardiovascular and total mortality among diabetic and non-diabetic individuals with or without history of myocardial infarction
}

Received: 13 August 2004 / Accepted: 6 January 2005 / Published online: 13 April 2005

(C) Springer-Verlag 2005

\begin{abstract}
Aims/hypothesis: We investigated the associations of type 2 diabetes and a history of myocardial infarction (MI) with coronary heart disease, stroke, cardiovascular and total mortality, and whether these effects are different in men and women. Methods: Study cohorts included 51,735 Finnish men and women aged $25-74$ years. Smoking status and the history of MI and type 2 diabetes were recorded, and height, weight, blood pressure and serum cholesterol levels were measured at baseline. Additional data on the occurrence of diabetes and MI prior to baseline survey were obtained from national hospital discharge and drug registers through computerised register linkage. Mortality data were obtained from the national mortality register. Results: During a mean follow-up period of 17.2 years, 9,201 deaths were recorded. Hazard ratios (HRs) for coronary mortality were 2.1, 4.0 and 6.4 among men with diabetes alone, men with MI alone, and men with diabetes and MI, respectively, compared with men with neither diabetes nor a history of MI. The corresponding HRs among women were 4.9, 2.5 and 9.4, respectively. The equivalent HRs for cardiovascular and total mortality were $2.2,3.3$ and 6.0 , and $1.8,2.3$ and 3.7 in men, and 4.4, 2.4 and 6.5, and 3.2, 1.7 and 4.4 in women, respectively. Diabetic men and women had similar total mortality rates, whereas total mortality
\end{abstract}

G. Hu $(\bowtie) \cdot$ P. Jousilahti $\cdot$ Q. Qiao $\cdot$ S. Katoh · J. Tuomilehto Diabetes and Genetic Epidemiology Unit,

Department of Epidemiology and Health Promotion,

National Public Health Institute,

Mannerheimintie 166,

00300 Helsinki, Finland

e-mail: hu.gang@ktl.fi

Tel.: +358-9-19127366

Fax: +358-9-19127313

G. Hu $\cdot$ P. Jousilahti $\cdot$ Q. Qiao $\cdot$ J. Tuomilehto

Department of Public Health, University of Helsinki,

Helsinki, Finland

S. Katoh

Department of Internal Medicine,

Jikei University School of Medicine,

Tokyo, Japan rates were markedly higher among men in the other three diabetes-MI categories. Conclusions/interpretation: Diabetes and MI markedly increase cardiovascular and all-cause mortality. In women, the association between diabetes and mortality was stronger than that between MI and mortality, whereas the converse was true among men.

Keywords Cardiovascular disease $\cdot$ Coronary heart disease - Gender difference - Myocardial infarction . Type 2 diabetes

Abbreviations CVD: cardiovascular disease . HR: hazard ratio - ICD: International Classification of Diseases · MI: myocardial infarction - WHO: World Health Organization

\section{Introduction}

Type 2 diabetes is one of the fastest growing public health problems worldwide because of increasing obesity and a sedentary lifestyle. The number of diabetic patients in the world has been estimated to at least double over the next 25-30 years [1, 2]. Cardiovascular disease (CVD), especially CHD and stroke, is the leading cause of death among patients with type 2 diabetes [3]. A Finnish cohort study that included men and women found that the CVD mortality rate among diabetic subjects without prior myocardial infarction (MI) was similar, or even higher than that in nondiabetic subjects with prior MI [4]. It is also known that women with diabetes will lose their relative protection (compared with men) against CVD [5]. However, only two previous studies have compared sex differences in the risk of CHD death associated with prevalent diabetes and established CHD [6, 7]. No previous study has investigated CHD, stroke, CVD and total mortality as separate endpoints. The aims of this study are to: (1) compare the independent and joint effects of diabetes and MI at baseline on CHD, stroke, CVD and all-cause mortality; and (2) examine the extent to which the independent associations of diabetes and MI with mortality differ between men and women. 


\section{Subjects and methods}

Subjects Six independent cross-sectional population surveys were carried out in the provinces of Kuopio and North Karelia in eastern Finland in 1972, 1977, 1982, 1987, 1992 and 1997 [8]. The survey was expanded to include the Turku-Loimaa region in southwestern Finland in 1982, the Helsinki capital area in 1992 and the northern province of Oulu in 1997. In 1972 and 1977, a random sample 6.6\% of the population born between 1913 and 1947 was selected. Since 1982, the sample chosen has been stratified by area, sex and 10-year age group according to the MONICA (Monitoring Trends and Determinants of Cardiovascular Disease) protocol of the World Health Organization (WHO) $[9,10]$. The subjects included in the six surveys were aged 25-64 years, and the 1997 survey also included subjects aged 65-74 years. Subjects who participated in more than one survey were only included in the first survey cohort. The total sample size of the six surveys was 53,166. The participation rate varied from 74 to $88 \%$ [8]. The final sample comprised 25,215 men and 26,520 women after excluding subjects with type 1 diabetes $(n=113)$ and subjects with incomplete data $(n=1,318)$. Subjects gave informed consent (verbal 1972-1992 and signed 1997) to participate in the survey. The surveys were conducted according to the ethics rules of the National Public Health Institute and the investigations were carried out in accordance with the Declaration of Helsinki.

Measurements A self-administered questionnaire was mailed to the participants in advance. It included questions about smoking and medical history. Based on the responses, the participants were classified as never, ex- and current smokers. At the study site, specially trained research nurses checked that the questionnaire was fully completed and measured blood pressure, height and weight using a standardised protocol [9]. Blood pressure was measured after 5 min of rest using a standard mercury sphygmomanometer; height was measured without shoes; and weight was measured with light clothing. BMI was calculated as weight in kilograms divided by the square of the height in metres. After blood pressure measurement, a venous blood specimen was drawn. In 1972 and 1977, total cholesterol concentrations were determined using the Lieberman-Burchard method, whereas an enzymatic method (CHOD-PAP; Boehringer Mannheim, Mannheim, Germany) was employed in the later surveys. Because the enzymatic method gave $2.4 \%$ lower values than the Lieberman-Burchard method, the 1972 and 1977 values were corrected by this percentage. All samples were analysed in the same laboratory at National Public Health Institute.

Assessment of diabetes MI at baseline Assessment of diabetes and MI status at baseline was based on self reporting and on the data supplied by two nationwide registers. Since 1968, the National Hospital Discharge Register has included diagnosis at hospital discharge. The validity of the data has been assessed and found to be generally good [11] Data on diabetes medication were obtained from the Social Insurance Institution of Finland's register on special reimbursement for glucose-lowering drugs. Glucose-lowering drugs prescribed by a physician are free of charge in Finland subject to the approval of the case history prepared by the treating physicians. The physician confirms the diagnosis of diabetes using the criteria recommended by the WHO [12]. All patients who receive medication free of charge (either oral glucose-lowering agents or insulin) are entered into a register maintained by the Social Insurance Institution of Finland.

Subjects who reported having diabetes on the questionnaire, who had been diagnosed with diabetes at hospital discharge, or who had been approved for medication for diabetes free of charge prior to the baseline survey were classified as having diabetes. Of the 1,108 subjects with diabetes, 402 were identified by the questionnaire alone, 51 by the hospital discharge diagnosis alone, 128 by approval for medication free of charge alone, seven by both the questionnaire and the hospital discharge diagnosis, 349 by both the questionnaire and approval for medication free of charge, 66 by both the hospital discharge diagnosis and approval for medication free of charge, and 105 by all three sources. The subjects with MI were those who reported

Table 1 Characteristics of the study sample according to history of diabetes and MI at baseline

\begin{tabular}{|c|c|c|c|c|c|c|c|c|}
\hline & \multicolumn{4}{|l|}{ Men } & \multicolumn{4}{|l|}{ Women } \\
\hline & $-\mathrm{DM}-\mathrm{MI}$ & $+\mathrm{DM}-\mathrm{MI}$ & $-\mathrm{DM}+\mathrm{MI}$ & $+\mathrm{DM}+\mathrm{MI}$ & $-\mathrm{DM}-\mathrm{MI}$ & $+\mathrm{DM}-\mathrm{MI}$ & $-\mathrm{DM}+\mathrm{MI}$ & $+\mathrm{DM}+\mathrm{MI}$ \\
\hline Number of participants & 23,638 & 496 & 982 & 99 & 25,681 & 466 & 326 & 47 \\
\hline Age (years) & $43.9 \pm 11.4$ & $53.0 \pm 10.7$ & $56.9 \pm 8.0$ & $58.7 \pm 8.3$ & $44.4 \pm 11.3$ & $52.4 \pm 11.0$ & $58.3 \pm 6.5$ & $60.1 \pm 7.0$ \\
\hline BMI $\left(\mathrm{kg} / \mathrm{m}^{2}\right)$ & $26.2 \pm 3.7$ & $28.2 \pm 4.4$ & $27.7 \pm 4.0$ & $29.3 \pm 4.4$ & $26.0 \pm 4.7$ & $29.7 \pm 6.1$ & $29.5 \pm 5.1$ & $31.1 \pm 5.3$ \\
\hline Diastolic BP (mmHg) & $88 \pm 12$ & $90 \pm 14$ & $89 \pm 12$ & $85 \pm 13$ & $84 \pm 12$ & $88 \pm 13$ & $90 \pm 12$ & $90 \pm 15$ \\
\hline Systolic BP (mmHg) & $143 \pm 19$ & $151 \pm 23$ & $147 \pm 22$ & $150 \pm 23$ & $139 \pm 23$ & $152 \pm 25$ & $153 \pm 23$ & $161 \pm 26$ \\
\hline Serum cholesterol (mmol/l) & $6.2 \pm 1.2$ & $6.2 \pm 1.3$ & $6.5 \pm 1.4$ & $6.3 \pm 1.5$ & $6.1 \pm 1.3$ & $6.3 \pm 1.3$ & $6.8 \pm 1.5$ & $6.7 \pm 1.7$ \\
\hline \multicolumn{9}{|l|}{ Smoking (\%) } \\
\hline Never & 33.9 & 31.5 & 16.8 & 19.2 & 74.7 & 79.2 & 78.5 & 78.7 \\
\hline Past & 23.3 & 33.7 & 43.6 & 48.5 & 8.0 & 7.3 & 10.4 & 8.5 \\
\hline Current & 42.8 & 34.9 & 39.6 & 32.3 & 17.3 & 13.5 & 11.1 & 12.8 \\
\hline
\end{tabular}

Data are means \pm SD or percentages

$D M$ Diabetes 
Table 2 Age-standardised CHD, stroke, CVD and total mortality rates of the study sample according to history of diabetes and MI at baseline

\begin{tabular}{|c|c|c|c|c|c|c|c|c|}
\hline \multirow[t]{2}{*}{ Statistics } & \multicolumn{4}{|l|}{ Men } & \multicolumn{4}{|l|}{ Women } \\
\hline & $-\mathrm{DM}-\mathrm{MI}$ & $+\mathrm{DM}-\mathrm{MI}$ & $-\mathrm{DM}+\mathrm{MI}$ & $+\mathrm{DM}+\mathrm{MI}$ & -DM-MI & $+\mathrm{DM}-\mathrm{MI}$ & $-\mathrm{DM}+\mathrm{MI}$ & $+\mathrm{DM}+\mathrm{MI}$ \\
\hline Number of participants & 23,638 & 496 & 982 & 99 & 25,681 & 466 & 326 & 47 \\
\hline \multicolumn{9}{|l|}{ Number of deaths } \\
\hline CHD & 1,665 & 85 & 320 & 42 & 783 & 74 & 53 & 17 \\
\hline Stroke & 413 & 30 & 27 & 12 & 377 & 30 & 26 & 2 \\
\hline CVD & 2,398 & 127 & 371 & 56 & 1,401 & 120 & 86 & 21 \\
\hline Total & 4,955 & 207 & 510 & 67 & 3,127 & 192 & 117 & 26 \\
\hline Person-years & 400,141 & 6,070 & 10,529 & 859 & 461,788 & 6,376 & 4,731 & 479 \\
\hline \multicolumn{9}{|c|}{ Mortality rate per 10,000 person-years ${ }^{a}$} \\
\hline $\mathrm{CHD}$ & 47 & 98 & 191 & 344 & 18 & 77 & 40 & 218 \\
\hline Stroke & 12 & 53 & 19 & 131 & 10 & 33 & 51 & 12 \\
\hline CVD & 68 & 163 & 224 & 482 & 34 & 127 & 97 & 241 \\
\hline Total & 143 & 300 & 305 & 579 & 77 & 220 & 164 & 270 \\
\hline
\end{tabular}

${ }^{a}$ Age-standardised mortality rates were calculated using a European standard population with 10-year age intervals $D M$ Diabetes

having MI on the questionnaire, or who had received a diagnosis of MI at hospital discharge prior to the baseline survey. Of the 1,454 subjects with MI, 858 were identified by the questionnaire alone, 79 by the hospital discharge diagnosis alone, and 517 by both sources.
Prospective follow-up Until the end of 2001 the study cohorts were followed through computerised register linkage using the personal identification number assigned to every resident in Finland. Mortality data were obtained from Statistics Finland (Helsinki, Finland). The International
Table 3 Hazard ratios $(95 \% \mathrm{CI})$ for CHD, stroke, CVD and total mortality according to history of diabetes and MI

Model 1 Adjusted for age and study year; Model 2 adjusted for age, study year, BMI, systolic $\mathrm{BP}$, total cholesterol and smoking; $D M$ diabetes

\begin{tabular}{|c|c|c|c|c|}
\hline & $-\mathrm{DM}-\mathrm{MI}$ & $+\mathrm{DM}-\mathrm{MI}$ & $-\mathrm{DM}+\mathrm{MI}$ & $+\mathrm{DM}+\mathrm{MI}$ \\
\hline \multicolumn{5}{|l|}{ Men } \\
\hline \multicolumn{5}{|c|}{ CHD mortality } \\
\hline Model 1 & 1.00 & $2.25(1.81-2.80)$ & $4.18(3.69-4.74)$ & $6.23(4.57-8.50)$ \\
\hline Model 2 & 1.00 & $2.11(1.70-2.63)$ & $3.97(3.50-4.50)$ & $6.41(4.69-8.76)$ \\
\hline \multicolumn{5}{|c|}{ Stroke mortality } \\
\hline Model 1 & 1.00 & $3.12(2.15-4.54)$ & $1.39(0.94-2.07)$ & $7.15(3.99-12.8)$ \\
\hline Model 2 & 1.00 & $2.66(1.82-3.88)$ & $1.42(0.95-2.11)$ & $7.35(4.08-13.3)$ \\
\hline \multicolumn{5}{|c|}{ CVD mortality } \\
\hline Model 1 & 1.00 & $2.33(1.95-2.79)$ & $3.36(3.00-3.77)$ & $5.80(4.44-7.59)$ \\
\hline Model 2 & 1.00 & $2.16(1.80-2.58)$ & $3.26(2.91-3.65)$ & $5.95(4.55-7.80)$ \\
\hline \multicolumn{5}{|c|}{ Total mortality } \\
\hline Model 1 & 1.00 & $1.89(1.64-2.17)$ & $2.28(2.08-2.51)$ & $3.48(2.73-4.44)$ \\
\hline Model 2 & 1.00 & $1.84(1.59-2.11)$ & $2.27(2.06-2.49)$ & $3.71(2.90-4.73)$ \\
\hline \multicolumn{5}{|l|}{ Women } \\
\hline \multicolumn{5}{|c|}{ CHD mortality } \\
\hline Model 1 & 1.00 & $5.06(3.98-6.45)$ & $2.49(1.88-3.30)$ & $13.8(8.45-22.5)$ \\
\hline Model 2 & 1.00 & $4.89(3.84-6.24)$ & $2.46(1.85-3.26)$ & $9.42(5.75-15.4)$ \\
\hline \multicolumn{5}{|c|}{ Stroke mortality } \\
\hline Model 1 & 1.00 & $4.38(3.01-6.39)$ & $2.85(1.90-4.26)$ & $3.39(0.84-13.7)$ \\
\hline Model 2 & 1.00 & $3.91(2.68-5.72)$ & $2.97(1.98-4.44)$ & $2.20(0.54-8.92)$ \\
\hline \multicolumn{5}{|c|}{ CVD mortality } \\
\hline Model 1 & 1.00 & $4.67(3.87-5.64)$ & $2.42(1.94-3.01)$ & $9.45(6.11-14.6)$ \\
\hline Model 2 & 1.00 & $4.35(3.60-5.26)$ & $2.43(1.95-3.04)$ & $6.46(4.16-10.0)$ \\
\hline \multicolumn{5}{|c|}{ Total mortality } \\
\hline Model 1 & 1.00 & $3.42(1.95-3.96)$ & $1.66(1.38-2.00)$ & $5.25(3.56-7.75)$ \\
\hline Model 2 & 1.00 & $3.21(2.77-3.72)$ & $1.66(1.38-2.00)$ & $4.35(2.94-6.42)$ \\
\hline
\end{tabular}


Table 4 Hazard ratios for CHD, stroke, CVD and total mortality in diabetic subjects without MI as compared with non-diabetic subjects with MI

\begin{tabular}{ccccc}
\hline & \multicolumn{2}{l}{ Hazard ratio $(95 \% \mathrm{CI})^{\mathrm{a}}$} & \multirow{2}{*}{$\chi^{2 \mathrm{c}}$} & \multirow{2}{*}{$p$ value $^{\mathrm{b}}$} \\
\cline { 2 - 4 } & Men & Women & & \\
\hline CHD mortality & 0.56 & 1.70 & 23.57 & $<0.001$ \\
& $(0.44-0.72)$ & $(1.17-2.47)$ & $(1 d f)$ & \\
Stroke mortality & 1.68 & 1.15 & 1.47 & $>0.1$ \\
& $(0.96-2.92)$ & $(0.66-2.01)$ & $(1 d f)$ & \\
Cardiovascular & 0.69 & 1.55 & 21.21 & $<0.001$ \\
mortality & $(0.56-0.86)$ & $(1.15-2.08)$ & $(1 d f)$ & \\
Total mortality & 0.82 & 1.80 & 29.50 & $<0.001$ \\
& $(0.69-0.97)$ & $(1.41-2.29)$ & $(1 d f)$ & \\
\hline
\end{tabular}

${ }^{a}$ Ratio for diabetic subjects without MI as compared with nondiabetic subjects with MI, adjusted for age, study year, BMI, systolic BP, total cholesterol and smoking

${ }^{\mathrm{b}}$ The interaction in terms of sex with diabetes or MI for mortality were tested using a log likelihood ratio test

Classification of Diseases 8 th, 9 th and 10th revisions (ICD8 , ICD-9, ICD-10, respectively) were used for coding the causes of death. ICD codes 390-459 (ICD-8 and ICD-9) and I00-I99 (ICD-10) were classified as CVD deaths, 410-414 (ICD-8 and ICD-9) and I20-I25 (ICD-10) as CHD deaths, and 430-438 (ICD-8 and ICD-9) and I60-I66 (ICD-10) as deaths caused by stroke.

Statistical analyses All data were analysed using SPSS for Windows, Version 11.5 (SPSS, Chicago, IL, USA). The sexspecific CHD, stroke, CVD and total mortality rates were calculated according to 10-year age intervals, and directly age-standardised rates were determined using a European standard population aged 25-74 years for each of the four groups at baseline [13]: neither diabetes nor MI (-DM-MI), diabetes alone (+DM-MI), MI alone $(-\mathrm{DM}+\mathrm{MI})$, and diabetes and MI $(+\mathrm{DM}+\mathrm{MI})$. Cox proportional hazards models were used to estimate the hazard ratios (HRs) for CHD, stroke, CVD and total mortality associated with diabetes and
MI status. The analyses were initially performed adjusting for age and study year; further adjustments were subsequently made for BMI, systolic blood pressure, total cholesterol and smoking at baseline. The analyses were carried out for men and women separately and for the two sexes combined to assess the joint association of diabetes, MI and sex with mortality. A log likelihood ratio test was carried out to test the significance of the interaction terms of sex with diabetes or MI for mortality. A $p$ value of less than 0.05 was considered to be statistically significant.

\section{Results}

The distribution of age and cardiovascular risk factors among the participants are presented in Table 1. During a mean follow-up period of 17.2 years, we identified 9,201 deaths from all causes, of which 3,039 deaths were coded as CHD, 917 as stroke, and 4,580 as CVD. As expected, the age-adjusted mortality rates for both sexes were lowest in subjects with neither MI nor diabetes, and highest in subjects with MI and diabetes at baseline (with the exception of stroke mortality in women, because of the low number of deaths in this subgroup) (Table 2). In men, a history of MI alone at baseline was associated with higher CHD, CVD and total mortality rates than diabetes alone; conversely, in women, higher mortality rates were associated with diabetes rather than with a history of MI.

Table 3 shows the multivariate-adjusted (age, study year, BMI, systolic blood pressure, cholesterol and smoking) HRs for CHD, stroke, CVD and total mortality in subjects with diabetes alone, in subjects with a history of MI alone, and in subjects with diabetes and MI as compared with subjects with neither diabetes nor MI. In general, in women, the association between diabetes and mortality was stronger than that between MI and mortality, whereas MI showed a relatively stronger association with mortality in men. However, compared with MI, diabetes showed a stronger association with stroke mortality in both sexes. In the analyses stratified by age group, the trends observed among subjects

Table 5 Hazard ratios (95\% CI) for CHD, stroke, CVD and total mortality according to history of diabetes and MI, with reference to women without diabetes or MI

\begin{tabular}{|c|c|c|c|c|c|c|c|c|}
\hline & \multicolumn{8}{|c|}{ Hazard ratio $(95 \% \text { confidence interval })^{\mathrm{a}}$} \\
\hline & \multicolumn{2}{|c|}{$-\mathrm{DM}-\mathrm{MI}$} & \multicolumn{2}{|l|}{$+\mathrm{DM}-\mathrm{MI}$} & \multicolumn{2}{|l|}{$-\mathrm{DM}+\mathrm{MI}$} & \multicolumn{2}{|l|}{$+\mathrm{DM}+\mathrm{MI}$} \\
\hline & Women & Men & Women & Men & Women & Men & Women & Men \\
\hline CHD mortality & 1.00 & $\begin{array}{l}2.85 \\
(2.57-3.17)\end{array}$ & $\begin{array}{l}4.78 \\
(3.76-6.08)\end{array}$ & $\begin{array}{l}5.89 \\
(4.67-7.43)\end{array}$ & $\begin{array}{l}2.59 \\
(1.96-3.43)\end{array}$ & $\begin{array}{l}11.31 \\
(9.74-13.14)\end{array}$ & $\begin{array}{l}8.96 \\
(5.52-14.55)\end{array}$ & $\begin{array}{l}18.35 \\
(13.31-25.29)\end{array}$ \\
\hline Stroke mortality & 1.00 & $\begin{array}{l}1.50 \\
(1.26-1.79)\end{array}$ & $\begin{array}{l}3.88 \\
(2.66-5.64)\end{array}$ & $\begin{array}{l}4.04 \\
(2.74-5.97)\end{array}$ & $\begin{array}{l}2.98 \\
(1.99-4.44)\end{array}$ & $\begin{array}{l}2.16 \\
(1.43-3.27)\end{array}$ & $\begin{array}{l}2.04 \\
(0.51-8.23)\end{array}$ & $\begin{array}{l}11.17 \\
(6.17-20.22)\end{array}$ \\
\hline Cardiovascular mortality & 1.00 & $\begin{array}{l}2.26 \\
(2.08-2.45)\end{array}$ & $\begin{array}{l}4.28 \\
(3.55-5.17)\end{array}$ & $\begin{array}{l}4.79 \\
(3.96-5.78)\end{array}$ & $\begin{array}{l}2.46 \\
(1.98-3.07)\end{array}$ & $\begin{array}{l}7.46 \\
(6.55-8.48)\end{array}$ & $\begin{array}{l}6.25 \\
(4.05-9.64)\end{array}$ & $\begin{array}{l}13.60 \\
(10.33-17.91)\end{array}$ \\
\hline Total mortality & 1.00 & $\begin{array}{l}1.78 \\
(1.68-1.88)\end{array}$ & $\begin{array}{l}3.20 \\
(2.77-3.71)\end{array}$ & $\begin{array}{l}3.22 \\
(2.78-3.72)\end{array}$ & $\begin{array}{l}1.69 \\
(1.40-2.03)\end{array}$ & $\begin{array}{l}4.05 \\
(3.66-4.49)\end{array}$ & $\begin{array}{l}4.31 \\
(2.92-6.34)\end{array}$ & $\begin{array}{l}6.58 \\
(5.14-8.42)\end{array}$ \\
\hline
\end{tabular}

${ }^{\mathrm{a}}$ Hazard ratios were adjusted for age, study year, BMI, systolic BP, total cholesterol and smoking $D M$ Diabetes 
aged $<50$ years and those aged $\geq 50$ years were almost the same (except for women aged $<50$ years who had diabetes and a history of MI; this subgroup only included two subjects).

Table 4 compares the associations between diabetes and mortality with those between a history of MI and mortality. The risks of CHD, CVD and total mortality were lower in men with diabetes alone than in men with a history of MI alone (HRs 0.56, 0.69 and 0.82, respectively). Conversely, women with diabetes alone had markedly higher risks of mortality than women with MI alone (HRs 1.70, 1.55 and 1.80 , respectively). These sex differences in hazard ratios were statistically significant for CHD mortality $\left(\chi^{2}=23.57\right.$, $1 d f, p<0.001)$, CVD mortality $\left(\chi^{2}=21.21,1 d f, p<0.001\right)$ and total mortality $\left(\chi^{2}=29.5,1 d f, p<0.001\right)$. In both men and women the relative risk of stroke mortality tended to be higher in subjects with diabetes alone than in subjects with MI alone ( $\mathrm{HR}=1.68,95 \%$ CI $0.96-2.92$ in men; $\mathrm{HR}=1.15$ $95 \%$ CI $0.66-2.01$ in women), and the stroke mortality rate was similar for the two sexes $\left(\chi^{2}=1.47,1 d f, p>0.1\right)$.

The associations between sex, diabetes and MI combined and cardiovascular and total mortality are presented in Table 5. Women with neither diabetes nor a history of MI were used as the reference group. Men with neither diabetes nor MI, men with MI alone, and men with diabetes and MI had markedly higher mortality rates (adjusted for other risk factors) than women with the same disease status. However, among diabetic subjects, the total mortality rate did not differ between men and women and the sex difference in CVD mortality was small.

\section{Discussion}

In our analysis we studied the associations of diabetes and MI with CHD, stroke, CVD and total mortality separately, and the sex difference in the risk of death. A history of MI and prevalent diabetes at baseline markedly increased the risk of CHD, stroke, CVD and total mortality in men and women. The associations of diabetes alone and diabetes and MI combined with mortality were relatively stronger in women, whereas MI showed a relatively stronger association with mortality in men. However, in both sexes, diabetes was more strongly associated with stroke mortality than MI was. Although diabetic men and women had similar multivariate-adjusted absolute mortality rates, men with neither diabetes nor a history of MI, men with MI alone, and men with diabetes and MI had markedly higher mortality rates than women in the same disease category.

Our main results are in keeping with the findings of most previous studies [4, 6, 7, 14-18]. However, our study is the first to compare the associations of prevalent diabetes and established CHD with CHD, CVD, stroke and total mortality as study endpoints in both sexes. The analyses of another Finnish cohort study that included men and women [4] and those of the Nurses' Health Study, which included women alone [14], suggested that the risk of CHD among subjects with a history of diabetes without prior MI was similar to that in non-diabetic subjects with prior MI. The analyses of the Framingham Study revealed that the risk of CHD mortality was higher in men with prior CHD than in men with prior diabetes [7]. However, this relationship was reversed in women, with prior diabetes being associated with a greater risk of CHD mortality [7]. These findings are consistent with our study. In the Hoorn Study, the risk of CVD events in women with a prior diagnosis of diabetes was similar to that in non-diabetic women with prior CVD, whereas non-diabetic men with prior CVD were found to be at a higher risk of CVD than men with a prior diagnosis of diabetes without prior CVD [6]. The results of the Health Professionals Follow-up Study [15] and the Multiple Risk Factor Intervention Trial, both of which included men alone [18], and those of a Scottish population-based study [16] and the Atherosclerosis Risk in Communities Study [17], both of which included men and women, reported that the incidence of CHD and mortality from CHD, CVD and all causes was lower in individuals with diabetes than in individuals with prior CHD. In the present study we also found that, in both sexes, the risk of death from stroke was higher among diabetic subjects than among those with a history of MI.

In the present study, the relative risks of death from CHD, CVD and all causes were higher in diabetic women than in diabetic men (relative to women and men without diabetes, respectively). Thus, our findings further support the hypothesis that the presence of diabetes reduces the usual female advantage over males regarding CVD risk [5]. The large differences observed between non-diabetic men and women in terms of the absolute CHD, CVD and total mortality were almost non-existent in diabetic patients. All these trends were identified regardless of MI status. Moreover, women with a history of diabetes were at a higher risk of CHD, stroke, CVD and total mortality than women with prior MI. The reason why diabetes confers a greater relative risk for death in women than in men is not clear. The marked increase in CVD risk in diabetic women could partly be explained by the loss of the women's usually less atherogenic lipid profile [19]. In addition, the results of the DECODE (Diabetes Epidemiology: Collaborative Analyses of Diagnostic Criteria in Europe) study suggested that the association between hyperglycaemia and other risk factors combined and CVD risk may be stronger in women than in men [5]. It has been reported that the pharmacological treatment of hypertension [20-23] and cholesterol reduction with a statin therapy [24-26] are efficient ways of preventing CHD in diabetic patients, and the majority of the patients who participated in these trials were female. While a more efficient control of hyperglycaemia and improvements in other CVD risk factors are important in all diabetic patients, it is possible that women with diabetes may particularly benefit from more intensive interventions aimed at reducing the risk of CVD.

Our study has the following strengths: (1) the number of participants is large and from a homogeneous population; (2) the mean follow-up period, 17.2 years, was sufficient to record a large number of CVD events; (3) the majority of subjects with type 1 diabetes were excluded from the analysis; and (4) due to the computerised data linkage of 
national mortality data, the endpoint data collection was practically complete. Our study is limited by the fact that we did not perform a baseline glucose tolerance test, and may therefore have missed some cases of asymptomatic or diet-treated diabetes. However, the clinical diagnosis of diabetes in the hospital discharge register included patients not using drugs, which should reduce this potential underdiagnosis. The study was also limited by the fact that we could not obtain data on drug treatment for diabetes, MI and other chronic diseases.

In conclusion, diabetes and MI markedly increased the risk of CVD mortality and total mortality. Diabetes showed a relatively stronger association with mortality in women, whereas a history of MI was associated with a higher risk of mortality in men. Even though men had markedly higher CVD mortality and total mortality rates than women, mortality rates did not differ markedly between sexes among diabetic subjects. In order to reduce the CVD mortality and total mortality rates, more active management of diabetes may be appropriate, particularly in women.

Acknowledgements This study was supported by grants from the Finnish Academy (grant nos. 46558, 53585, 204274 and 205657). S. Katoh's work is supported by a grant from the Uehara Memorial Foundation in Japan. The authors have no relevant financial interest in this article.

\section{References}

1. King H, Aubert RE, Herman WH (1998) Global burden of diabetes, 1995-2025: prevalence, numerical estimates, and projections. Diabetes Care 21:1414-1431

2. Wild S, Roglic G, Green A, Sicree R, King H (2004) Global prevalence of diabetes: estimates for the year 2000 and projections for 2030. Diabetes Care 27:1047-1053

3. Muller WA (1998) Diabetes mellitus - long time survival. J Insur Med 30:17-27

4. Haffner SM, Lehto S, Ronnemaa T, Pyorala K, Laakso M (1998) Mortality from coronary heart disease in subjects with type 2 diabetes and in nondiabetic subjects with and without prior myocardial infarction. N Engl J Med 339:229-234

5. DECODE Study Group (2003) Gender difference in all-cause and cardiovascular mortality related to hyperglycaemia and newly-diagnosed diabetes. Diabetologia 46:608-617

6. Becker A, Bos G, de Vegt F et al (2003) Cardiovascular events in type 2 diabetes: comparison with nondiabetic individuals without and with prior cardiovascular disease: 10-year followup of the Hoorn Study. Eur Heart J 24:1406-1413

7. Natarajan S, Liao Y, Cao G, Lipsitz SR, McGee DL (2003) Sex differences in risk for coronary heart disease mortality associated with diabetes and established coronary heart disease. Arch Intern Med 163:1735-1740

8. Vartiainen E, Jousilahti P, Alfthan G, Sundvall J, Pietinen P, Puska P (2000) Cardiovascular risk factor changes in Finland, 1972-1997. Int J Epidemiol 29:49-56

9. World Health Organization MONICA Project Principal Investigators (1988) The World Health Organization MONICA Project (monitoring trends and determinants in cardiovascular disease): a major international collaboration. J Clin Epidemiol 41:105-114

10. Pajak A, Kuulasmaa K, Tuomilehto J, Ruokokoski E (1988) Geographical variation in the major risk factors of coronary heart disease in men and women aged 35-64 years: the WHO MONICA project. World Health Stat Q 41:115-140
11. Mahonen M, Salomaa V, Brommels M et al (1997) The validity of hospital discharge register data on coronary heart disease in Finland. Eur J Epidemiol 13:403-415

12. World Health Organization Study Group on Diabetes Mellitus (1985) Diabetes mellitus: report of a WHO study group. WHO Technical Report Series No. 727. World Health Organization, Geneva

13. Waterhouse J, Muir C, Correa P, Powell J (1976) Cancer incidence in five continents. IARC Scientific Publications, Lyon

14. Hu FB, Stampfer MJ, Solomon CG et al (2001) The impact of diabetes mellitus on mortality from all causes and coronary heart disease in women: 20 years of follow-up. Arch Intern Med $161: 1717-1723$

15. Lotufo PA, Gaziano JM, Chae CU et al (2001) Diabetes and allcause and coronary heart disease mortality among US male physicians. Arch Intern Med 161:242-247

16. Evans JM, Wang J, Morris AD (2002) Comparison of cardiovascular risk between patients with type 2 diabetes and those who had had a myocardial infarction: cross-sectional and cohort studies. BMJ 324:939-942

17. Lee CD, Folsom AR, Pankow JS, Brancati FL (2004) Cardiovascular events in diabetic and nondiabetic adults with or without history of myocardial infarction. Circulation 109:855860

18. Vaccaro O, Eberly LE, Neaton JD, Yang L, Riccardi G, Stamler J (2004) Impact of diabetes and previous myocardial infarction on long-term survival: 25 -year mortality follow-up of primary screenees of the Multiple Risk Factor Intervention Trial. Arch Intern Med 164:1438-1443

19. Siegel RD, Cupples A, Schaefer EJ, Wilson PW (1996) Lipoproteins, apolipoproteins, and low-density lipoprotein size among diabetics in the Framingham offspring study. Metabolism 45:1267-1272

20. Curb JD, Pressel SL, Cutler JA et al (1996) Effect of diureticbased antihypertensive treatment on cardiovascular disease risk in older diabetic patients with isolated systolic hypertension. Systolic Hypertension in the Elderly Program Cooperative Research Group. JAMA 276:1886-1892

21. UK Prospective Diabetes Study Group (1998) Tight blood pressure control and risk of macrovascular and microvascular complications in type 2 diabetes: UKPDS 38. BMJ 317:703-713

22. Tuomilehto J, Rastenyte D, Birkenhager WH et al (1999) Effects of calcium-channel blockade in older patients with diabetes and systolic hypertension. Systolic Hypertension in Europe Trial Investigators. N Engl J Med 340:677-684

23. The ALLHAT Officers and Coordinators for the Collaborative Research Group (2002) Major outcomes in high-risk hypertensive patients randomized to angiotensin-converting enzyme inhibitor or calcium channel blocker vs diuretic: the antihypertensive and lipid-lowering treatment to prevent heart attack trial (ALLHAT). JAMA 288:2981-2997

24. Pyorala K, Pedersen TR, Kjekshus J, Faergeman O, Olsson AG, Thorgeirsson G (1997) Cholesterol lowering with simvastatin improves prognosis of diabetic patients with coronary heart disease: a subgroup analysis of the Scandinavian simvastatin survival study (4S). Diabetes Care 20:614-620

25. Heart Protection Study Collaborative Group (2002) MRC/BHF heart protection study of cholesterol lowering with simvastatin in 20,536 high-risk individuals: a randomised placebo-controlled trial. Lancet 360:7-22

26. Goldberg RB, Mellies MJ, Sacks FM et al (1998) Cardiovascular events and their reduction with pravastatin in diabetic and glucose-intolerant myocardial infarction survivors with average cholesterol levels: subgroup analyses in the cholesterol and recurrent events (CARE) trial. The Care Investigators. Circulation 98:2513-2519 